\title{
Recent updates in the therapeutic management of patients with co- infection of COVID-19 with other seasonal epidemics prone disease
}

\section{Gedam D. ${ }^{1 *}$, Verma M. ${ }^{2}$}

DOI: https://doi.org/10.17511/ijphr.2020.i05.01

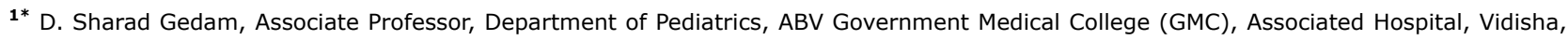
Madhya Pradesh, India.
}

2 Mamta Verma, Associate Professor, College of Nursing, All India Institute of Medical Sciences, Bhopal, Madhya Pradesh, India.

COVID 19 Pandemic is most significant disease in last 100 years. It is now becoming challenging to control the Pandemic in different part of world. Some countries are facing $2^{\text {nd }} \& 3^{\text {rd }}$ wave of severe disease with no evidences of herd immunity in most of European and North American countries. It become more challenging to treat the already co-existing morbidities, seasonal diseases along with COVID 19.

Keywords: COVID 19, Co-morbidities, Seasonal epidemics

Corresponding Author

D. Sharad Gedam, Associate Professor, Department of Pediatrics, ABV Government Medical College (GMC), Associated Hospital, Vidisha, Madhya Pradesh, India.

Email: sharad.gedam@gmail.com

\section{How to Cite this Article}

Gedam DS, Verma M. Recent updates in the therapeutic management of patients with coinfection of COVID-19 with other seasonal epidemics prone disease. Public Health Rev Int J Public Health Res. $2020 ; 7(5): 35-37$.

Available From

https://publichealth.medresearch.in/index.php/ijphr/ To Browse

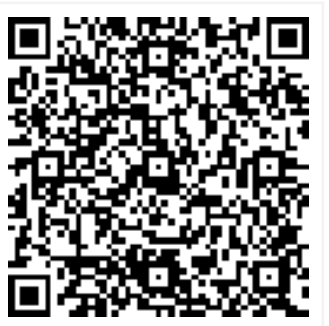
article/view/144

Manuscript Received Conflict of Interest No
Review Round 2

Ethical Approval Yes
Review Round 3

Plagiarism X-checker $6 \%$
Accepted $\underset{\text { Nil }}{\text { Funding }}$

re Societ Oparad Gedam, Mamta Verma and Published by Siddharth Health Research and Social Welfare Soci
Open Access article licensed under a Creative Commons Attribution 4.0 International License https://creativecommons.org/licenses/by/4.0/ unported [CC BY 4.0]. 
Almost all States/UTs of the country are affected by COVID-19. Given the migratory model of epidemic predisposed to diseases witnessed every year in our country, it diseases like Dengue, Malaria, Seasonal Influenza, Leptospirosis, Chikungunya, Enteric fever, etc. can not only present as a diagnostic predicament but may co-exist in COVID cases.

This presents questions in clinical and laboratory diagnosis of COVID and has an impact on clinical management and patient consequences. As per the World Health Organization (WHO) case definition, a COVID case may present with:

- Acute onset of fever AND cough; OR

- Acute onset of ANY THREE OR MORE of the following signs or symptoms: fever, cough, general weakness/fatigue, headache, myalgia, sore throat, coryza, dyspnoea, anorexia/nausea/ vomiting, diarrhea, altered mental status.

This case definition, even though vulnerable, is not very particular. Seasonal epidemic-prone diseases may all present as a febrile illness, with symptoms that mimic COVID-19.

If there is a co-infection, then apart from the febrile illness there may be a constellation of signs and symptoms that may lead to difficulty in diagnosis.

Method to the diagnosis of suspected coinfection: $A$ high index of suspicion must be maintained for epidemic-prone diseases (e.g. Dengue, Malaria, Chikungunya, Seasonal influenza, Leptospirosis) prevalent in a particular geographic region during monsoon and post-monsoon seasons.

Bacterial co-infections must also be suspected in moderate or severe cases of COVID-19 not responding to treatment.

- Malaria/Dengue: It must be borne in mind that malaria/dengue can coexist with other infections, and thus confirmation of malaria/dengue infection does not rule out the possibility of the patient not suffering from COVID-19.

Similarly, a high index of suspicion of malaria/dengue must be there when a fever case is diagnosed as COVID-19, particularly during the rainy and post rainy season in areas endemic for these diseases.

Seasonal Influenza: Both COVID-19 and Seasonal Influenza present as Influenza-Like Illness (ILI)/SARI, hence all ILI/SARI cases in areas
Reporting COVID-19 cases must be evaluated and tested for both COVID-19 and Seasonal Influenza if both viruses are circulating in the population under consideration.

- Chikungunya: Chikungunya presents with acute onset of moderate to high-grade continuous fever and malaise followed by a rash, myalgia, and arthralgia. Respiratory failure may ensue in the late stages. Co-infection with COVID-19 may be suspected in Chikungunya endemic areas, in the months of monsoon.

- Leptospirosis: Leptospirosis apart from it presenting as a febrile illness, has also the tendency to manifest as acute respiratory illness, leading to respiratory distress and shock. In areas where Leptospirosis is known to cause outbreaks during monsoon/ postmonsoon, the possibility of coinfection should be considered.

- Scrub Typhus: Scrub typhus is known to be prevalent in foothills of Himalayas viz Jammu \& Kashmir, Himachal Pradesh, Sikkim, Manipur, Nagaland, Meghalaya, etc. However, in the recent past, scrub typhus outbreaks have also been reported from Delhi, Haryana, Rajasthan, Maharashtra, Uttarakhand, Chhattisgarh, Tamil Nadu, and Kerala.

The clinical picture consists of sudden highgrade fever, severe headache, apathy, myalgia, and generalized lymphadenopathy. A maculopapular rash may appear first on the trunk and then on the extremities and blenches within a few days. The patients may develop complications that include interstitial pneumonia (30 to $65 \%$ of cases), meningoencephalitis, and myocarditis. Scrub typhus infection may co-exist with COVID-19.

Bacterial infections: Few patients with COVID-19 experience a secondary bacterial infection. In such cases, empiric antibiotic therapy as per local antibiogram needs to be considered. Despite the possibility of the above-mentioned co-infections, in present times of the pandemic, the approach to diagnosis for COVID-19 essentially remains the same. Testing protocol as per MoHFW/ICMR guidelines will be followed. However, besides, further tests for a likely co-infection will also be undertaken, whenever suspected.

Diagnostics While each of these infections is antigenically distinct with specific serological responses, yet in the eventuality of co-infections, 
Cross-reactions (resulting in false-positive /falsenegative results) cannot be ruled out, especially if the testing kits used are not having requisite sensitivity and specificity.

Hence the tests recommended by ICMR (for COVID19) and that recommended by the concerned program divisions (NVBDCP for vector-borne diseases [Malaria, Dengue, Chikungunya]) and NCDC (Seasonal Influenza, Leptospirosis, Scrub Typhus)] needs to be followed. Availability of rapid diagnostic kits for malaria, dengue, scrub typhus should be ensured in such COVID treatment facilities $[1,2]$.

Points related to specific therapeutic options and their use in cases with co-infection:

- Fluid Therapy: Fluid therapy to be given in coinfection cases depends on the hemodynamic status of the patient and degree of severity. One may follow national guidelines for clinical management of dengue fever for most coinfection cases. It is only in the presence of SARI with COVID-19 that we need to be careful with aggressive fluid administration as it leads to worsening of oxygenation. Close clinical monitoring of fluid status is required in such cases. Aggressive fluid resuscitation is recommended for COVID-19 patients in shock for initial resuscitation.

- LMWH: LMWH is being used and has been included in the National guidelines for the management of moderate to severe covid-19 cases as it is associated with increased thrombosis. Once the platelet count decreases to less than 1 lakh we need to be very careful with the use of LMWH and it may be withheld based on the clinical condition of the patient. The decision to administer LMWH and the dosage for the same should be based on close monitoring with D-dimer measurements. In any case of co-infection with active bleeding, LMWH needs to be stopped immediately.

- Use of Corticosteroids: Steroids specially Dexamethasone have recently been shown to be effective in severe covid-19 cases and have been recommended for the same. Dengue is a viral illness, it's course won't be affected much. Hence, the use of steroids can be continued as per COVID-19 management guidelines.

- Tocilizumab: To be used as per national management guidelines for COVID-19 management.
- Antivirals: To be used as per COVID-19 management guidelines.

Other supportive management to be continued as per the current guidelines.

\section{Reference}

01. Government of India Ministry of Health \& Family Welfare Directorate General of Health Services (EMR Division) Guidelines for the management of co-infection of COVID-19 with other seasonal epidemic-prone diseases.

Available at: [Article] [Crossref]

02. National Centre for Disease Control, Directorate General of Health Services, Ministry of Health and Family Welfare, Government of India, January 2020.

Available at: [Article] [Crossref] 